\title{
Systemic Therapy of Metastatic Breast Cancer: The Truth Beyond the Clinical Trials
}

\author{
Uwe Gueth $^{\mathrm{a}}$ Dorothy Jane Huang ${ }^{\mathrm{a}}$ Andreas Schoetzau ${ }^{\mathrm{b}}$ Wolfgang Holzgreve ${ }^{\mathrm{a}}$ \\ Edward Wight ${ }^{a}$

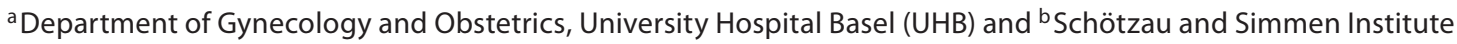 \\ for Biomathematics, Basel, Switzerland
}

\section{Key Words}

Breast cancer • Distant metastases - Systemic therapy

Chemotherapy $\cdot$ Endocrine therapy

\begin{abstract}
Objective: To depict a clear and coherent picture of the overall course of palliative treatment in an unselected study cohort over the course of time. Methods: We compared therapy type and course of 242 women whose distant metastatic disease was diagnosed from 1990 to 2006 and who ultimately died of the disease. We divided the patients into two subgroups depending on the year of diagnosis of metastases (group A: 1998-2006 vs. group B: 1990-1997). Results: In both subgroups, there were no significant differences in the general type of treatment and the number of administered therapy lines (no systemic therapy: 12.9 vs.13.7\%, $p=0.848$; endocrine therapy only: 20.4 vs. $25.2 \%, p=0.430$; chemotherapy only: 18.4 vs. $16.9 \%, p=0.735$; sequential combination regimen including endocrine therapy/chemotherapy/ trastuzumab: 46.9 vs. $44.2 \%, p=0.694$; median: 2 lines). In the cases where chemotherapy was administered, there were no differences between the number of lines among older and younger patients (median: two lines; $\geq 70$ years vs. $<70$ years: $p=0.269$ ). The median metastatic disease-specific survival increased from 16 months in the period from
\end{abstract}

1990 to 1997, to 21 months in the period from 1998 to 2000 $(p=0.062)$. Conclusion: The number of patients who died from metastatic breast cancer without receiving any antineoplastic therapy was surprisingly high. The use of newer agents and regimens in the treatment of metastatic breast cancer was associated with an improved survival over time. Chemotherapy is a feasible option also among older patients.

Copyright $\odot 2009$ S. Karger AG, Basel

\section{Introduction}

There exists a vast amount of published clinical trials concerning the systemic therapy of metastatic breast cancer [overview in 1-6]. Nearly all trials, however, concentrate only on the feasibility and impact of defined therapy options. They focus on the evaluation and comparison of particular antineoplastic agents and drugs, but in doing so, can only evaluate particular therapy options in preselected groups of patients in certain situations and at a certain point of time. Clinical trials which evaluate patients' outcome in regard to specific treatments do not usually consider the subsequent therapy course of the disease when the study period is finished. However, an analysis of the entire course of therapy of patients with

Uwe Gueth, MD

Department of Gynecology and Obstetrics, University Hospital Basel Spitalstrasse 21

CH-4031 Basel (Switzerland)

Tel. +41 61265 9028, Fax +4161265 9037, E-Mail ugueth@uhbs.ch 
metastatic breast cancer shows that these patients receive a multitude of therapy lines up until death. These subsequent therapies are hardly considered in the analysis of trial data. Thus, a weak point of these studies is that their ability to describe the overall course of recurrent disease is limited.

The goal of this study was to depict a clear and coherent picture of the overall palliative treatment setting and to demonstrate its changes and developments over time. We did not simply demonstrate specific treatments (this is the domain of clinical trials), but rather complete courses of therapy in an unselected group of patients (this is not addressed by clinical trials) and by doing so we wanted to demonstrate the truth beyond the clinical trials.

\section{Patients and Methods}

The Basel Breast Cancer Data Base is comprised of extensive data concerning clinical, histo- and pathomorphologic features and treatment characteristics of all patients whose primary invasive breast carcinoma was diagnosed and treated at the University Women's Hospital Basel (Basel, Switzerland) since 1990. For this study, we analyzed the data of 1,187 patients who were initially diagnosed up to 2006. The patients were followed until death or, if they remained alive and disease free, for a maximum of 15 years. They were seen for a maximum of 6 months before conclusion of the data collection in December 2007. With the exception of 37 patients (3.1\% of the entire study group) who were lost to follow-up, we could provide outcome information for all other patients. Of these, 301 women already had distant metastases at initial diagnosis (stage IV; $\mathrm{n}=67,5.6 \%$ ) or developed metastases in the course of time $(\mathrm{n}=234,19.7 \%)$. The data regarding the course of palliative therapy and outcome of these patients form the basis of this study. From these 301 patients, we could obtain information regarding the time of diagnosis of metastatic disease and date of death but we did not have complete information about the therapy course in the palliative situation for 4 patients. Thus, these were not considered for further analysis, and ultimately 297 patients were included in the study.

It was the goal of our study to give a general overview regarding the actual administered therapies in the systemic treatment of metastatic breast cancer. We compared the therapy course and outcome of women whose metastatic disease was diagnosed before 1998 with those whose distant metastases were diagnosed after 1998. In order to create homogeneous study groups, we analyzed only the 242 patients who ultimately died of their metastatic disease. In other words, we analyzed only completed treatment courses. Patients who died of other causes $(n=18)$ or were still alive at the conclusion of the observation period (i.e. whose therapies were presumably still ongoing, $n=37$ ) were excluded. For the purpose of our study, we listed the number of therapy lines and noted the general type of therapy: (1) no systemic treatment, (2) endocrine therapy only, (3) chemotherapy only, (4) trastuzum$\mathrm{ab}$ only, and (5) combination of the therapy types during the course of treatment.
Any change in the therapy regimen, i.e. the agent or combination of agents used, was viewed as a new line of treatment. The study design and data collection methods were approved by our institutional review board.

Statistical Analysis

To predict the survival with distant metastatic disease, we used the Kaplan-Meier method. Survival was defined as the interval from the date of diagnosis of distant metastases to the date of breast cancer-related death. Non-breast-cancer-related deaths were censored in the statistical analyses according to the same method used for patients who were alive. In this manner, a metastatic disease-specific survival (MDSS) was calculated. Statistical differences between groups in terms of survival curves were analyzed using the log rank test. To compare ordinal variables (number of therapy lines) between the two groups, the nonparametric exact Wilcoxon test was performed. Comparisons between nominal parameters were made with the Fisher exact test. $\mathrm{p}<0.05$ was considered significant. Statistical analyses were performed with R Development Core Team software, version 2.5.0 (Vienna, Austria).

\section{Results}

The clinicopathologic and outcome characteristics of the 297 patients with metastatic breast cancer included in the study are summarized in table 1 . The majority of the patients died of the disease $(\mathrm{n}=242,81.5 \%)$. The median follow-up time of patients who were alive was 39 months (range: 10-172 months). In 3 patients, we observed an asymptomatic long-term survival of more than 12 years.

Table 2 lists the general type of treatment in the 242 patients who died of metastatic breast cancer (i.e. completed therapy courses). Between patients who were initially diagnosed with metastatic disease between 1990 and 1997 and those who were diagnosed from 1998 to 2006, there was a significant difference regarding age ( 60.3 vs. 64.7 years, $p=0.0219$ ). There were no significant differences in the general type of treatment (no systemic therapy, $\mathrm{p}=0.848$; endocrine therapy only, $\mathrm{p}=0.430$; chemotherapy only, $\mathrm{p}=0.735$; combination regimen including endocrine therapy and/or chemotherapy and/or trastuzumab, $p=0.694$ ). Furthermore, the number of administered therapy lines did not differ significantly between the two groups.

Table 3 shows the general type of palliative treatment received by patients dependent on age at initial diagnosis of distant metastasis. Even in the subgroups comprised of patients younger than 70 years, approximately $10 \%$ of patients did not receive any antineoplastic palliative treatment ( $\leq 59$ years: $10.8 \%, 60-69$ years: $9.1 \%$ ). Among older patients ( $\geq 70$ years), the percentage of untreated pa- 
Table 1. Clinicopathologic characteristics of 297 patients with distant metastatic breast cancer

\begin{tabular}{|c|c|c|}
\hline Variable & Group A & Group B \\
\hline Entire group & $191(100)$ & $106(100)$ \\
\hline Stage IV at initial diagnosis & $46(24.1)$ & $20(18.9)$ \\
\hline DM in the course of time & $145(75.9)$ & $86(81.1)$ \\
\hline \multicolumn{3}{|l|}{ Median time from initial diagnosis to } \\
\hline diagnosis of DM (range), months & $44.5(2-166)$ & $25.5(2-100)$ \\
\hline \multicolumn{3}{|c|}{ Age at diagnosis of DM } \\
\hline Mean (range), years & $64.7(28-92)$ & $60.3(37-87)$ \\
\hline$\leq 59$ years & $59(30.9)$ & $53(50.0)$ \\
\hline $60-69$ years & $53(27.7)$ & $24(22.6)$ \\
\hline$\geq 70$ years & $79(41.4)$ & $29(27.4)$ \\
\hline \multicolumn{3}{|l|}{ TNM stage $^{1}$ at initial diagnosis } \\
\hline $\mathrm{I}$ & $18(9.4)$ & $8(7.6)$ \\
\hline II & $61(31.9)$ & $38(35.8)$ \\
\hline III & $66(34.6)$ & $40(37.7)$ \\
\hline \multicolumn{3}{|l|}{ Hormonal receptor status } \\
\hline Not known & $1(0.5)$ & $17(16.0)$ \\
\hline $\mathrm{ER}+/ \mathrm{PR}+$ & $101(52.9)$ & $52(49.1)$ \\
\hline $\mathrm{ER}+/ \mathrm{PR}-$ & $38(19.9)$ & $16(15.1)$ \\
\hline $\mathrm{ER}-/ \mathrm{PR}+$ & $3(1.6)$ & $2(1.9)$ \\
\hline $\mathrm{ER}-/ \mathrm{PR}-$ & $48(25.1)$ & $19(17.9)$ \\
\hline \multicolumn{3}{|l|}{ Grading } \\
\hline G1: well differentiated & $4(2.1)$ & - \\
\hline G2: moderately differentiated & $67(35.1)$ & $16(15.1)$ \\
\hline G3: poorly differentiated & $110(57.6)$ & $75(70.8)$ \\
\hline Not known/not applicable & $10(5.2)$ & $15(14.1)$ \\
\hline \multicolumn{3}{|l|}{ HER-2 neu status } \\
\hline Known & $151(79.1)$ & $20(18.9)$ \\
\hline Positive & $36(23.8)$ & $7(35.0)$ \\
\hline \multicolumn{3}{|l|}{ Adjuvant therapy } \\
\hline No therapy & $25(17.2)^{\mathrm{a}}$ & $24(27.9)^{\mathrm{a}}$ \\
\hline Endocrine therapy & $93(64.1)^{\mathrm{b}}$ & $50(58.1)^{\mathrm{b}}$ \\
\hline Chemotherapy & $66(45.5)^{c}$ & $19(22.1)^{c}$ \\
\hline \multicolumn{3}{|c|}{ Metastatic site at initial time of diagnosis of metastatic disease } \\
\hline Bone metastases only & $56(29.3)$ & $31(29.2)$ \\
\hline Visceral metastases & $135(70.7)$ & $75(70.8)$ \\
\hline \multicolumn{3}{|l|}{ Outcome status } \\
\hline Died of metastatic disease & $147(77.0)$ & $95(89.6)$ \\
\hline Died of other causes & $10(5.2)$ & $8(7.5)$ \\
\hline Alive & $34(17.8)$ & $3(2.8)$ \\
\hline
\end{tabular}

Unless otherwise indicated figures represent number with the percentage in parentheses. Group A = Distant metastases first diagnosed in 1998-2006; group B = distant metastases first diagnosed in 1990-1997; DM = distant metastases; ER = estrogen receptor; $\mathrm{PR}=$ progesterone receptor.

${ }^{\mathrm{a}} \mathrm{p}=0.0493 ;{ }^{\mathrm{b}} \mathrm{p}=0.4016 ;{ }^{\mathrm{c}} \mathrm{p}=0.0004$.

${ }^{1}$ AJCC (American Joint Committee on Cancer)/UICC (International Union Against Cancer) TNM Classification.
Table 2. Palliative treatment setting of 242 patients who died of metastatic breast cancer

\begin{tabular}{|c|c|c|}
\hline Variable & Group A & Group B \\
\hline Entire group & $147(100)$ & $95(100)$ \\
\hline No systemic therapy & $19(12.9)$ & $13(13.7)$ \\
\hline Mean age (range), years & $72.0(50-91)$ & $63.0(44-81)$ \\
\hline ET only & $30(20.4)$ & $24(25.2)$ \\
\hline Mean age (range), years & $72.7(53-86)$ & $66.2(44-87)$ \\
\hline 1 therapy line & $22(15.0)$ & $19(20.0)$ \\
\hline 2 therapy lines & $8(5.4)$ & $4(4.2)$ \\
\hline 3 therapy lines & - & $1(1.0)$ \\
\hline Median number of therapy lines & 1 & 1 \\
\hline CT only & $27(18.4)$ & $16(16.9)$ \\
\hline Mean age (range), years & $60.5(32-84)$ & $57.8(37-82)$ \\
\hline 1 therapy line & $11(7.5)$ & $7(7.4)$ \\
\hline 2 therapy lines & $10(6.8)$ & $3(3.2)$ \\
\hline 3 therapy lines & $3(2.0)$ & $5(5.3)$ \\
\hline 4 therapy lines & $2(1.4)$ & $1(1.0)$ \\
\hline $5-7$ therapy lines & $1(0.7)$ & - \\
\hline Median number of therapy lines & 2 & 2 \\
\hline Trastuzumab only & $2(1.4)$ & - \\
\hline Age range, years & $53-92$ & - \\
\hline Combination regimens & $69(46.9)$ & $42(44.2)$ \\
\hline Combination: CT and ET & $48(32.7)$ & $39(41.1)$ \\
\hline Mean age (range), years & $62.8(39-87)$ & $58.7(37-82)$ \\
\hline 2 therapy lines & $6(4.1)$ & $8(8.4)$ \\
\hline 3 therapy lines & $10(6.8)$ & $9(9.5)$ \\
\hline 4 therapy lines & $10(6.8)$ & $9(9.5)$ \\
\hline $5-6$ therapy lines & $21(14.3)$ & $11(11.6)$ \\
\hline $7-8$ therapy lines & $1(0.7)$ & $2(2.1)$ \\
\hline Median number of therapy lines & 4 & 3 \\
\hline \multicolumn{3}{|l|}{ Combination: } \\
\hline $\mathrm{CT}$ and trastuzumab & $13(8.8)$ & $1(1.0)$ \\
\hline Mean age (range), years & $52.5(28-80)$ & 70 \\
\hline 1 therapy line & $1(0.7)$ & - \\
\hline $2-3$ therapy lines & $5(3.4)$ & - \\
\hline 4-5 therapy lines & $5(3.4)$ & 1 \\
\hline $6-7$ therapy lines & $2(2.0)$ & - \\
\hline Median number of therapy lines & 3.5 & \\
\hline \multicolumn{3}{|l|}{ Combination: CT and ET } \\
\hline and trastuzumab & $8(5.4)$ & $2(2.1)$ \\
\hline Mean age (range), years & $56.6(37-70)$ & $43.5(42,51)$ \\
\hline $4-6$ therapy lines & $7(4.7)$ & $1(1.0)$ \\
\hline 9 therapy lines & $1(0.7)$ & - \\
\hline 10 therapy lines & - & $1(1.0)$ \\
\hline Median number of therapy lines & 5.5 & 8 \\
\hline
\end{tabular}

Unless otherwise indicated figures represent number with the percentage in parentheses. Group $\mathrm{A}=$ Distant metastases first diagnosed in 1998-2006; group B = distant metastases first diagnosed in 1990-1997; CT = chemotherapy; ET = endocrine therapy. 
Table 3. Type of palliative therapy among patients of different age groups who died of metastatic breast cancer

\begin{tabular}{lc}
\hline Entire group & $242(100)$ \\
\hline (1) $\leq 59$ years & $93(38.4)$ \\
No therapy & $10(10.8)$ \\
ET only & $11(11.8)$ \\
CT only & $22(23.7)$ \\
Combination: CT and/or ET and/or trastuzumab & $50(53.7)$ \\
Median number of CT lines & 2 \\
\hline (2) $60-69$ years & $66(27.3)$ \\
No therapy & $6(9.1)$ \\
ET only & $13(19.7)$ \\
CT only & $11(16.7)$ \\
Combination: CT and/or ET and/or trastuzumab & $36(54.5)$ \\
Median number of CT lines & 2 \\
\hline (3) 70 years & $83(34.3)$ \\
No therapy & $16(19.3)$ \\
ET only & $30(36.1)$ \\
CT only & $12(14.5)$ \\
Combination: CT and/or ET and/or trastuzumab & $25(30.1)$ \\
Median number of CT lines & 2
\end{tabular}

Unless otherwise indicated figures represent number with the percentage in parentheses. $\mathrm{ET}=$ Endocrine therapy; $\mathrm{CT}=$ chemotherapy.

${ }^{1}$ In one case, trastuzumab only.

Table 4. Number of chemotherapy lines received by patients who died of metastatic breast cancer in a current palliative setting (diagnosis of metastases after 1998)

$\begin{array}{lc}\begin{array}{l}\text { Total of patients who } \\ \text { received chemotherapy }\end{array} & 96(100) \\ 1 \text { therapy line } & 26(27.1) \\ 2 \text { therapy lines } & 31(32.3) \\ 3 \text { therapy lines } & 14(14.6) \\ 4 \text { therapy lines } & 14(14.6) \\ 5 \text { therapy lines } & 7(7.3) \\ 6 \text { therapy lines } & 1(1.0) \\ 7 \text { therapy lines } & 3(3.1)\end{array}$

Figures represent number with the percentage in parentheses.

tients increased to $19.3 \%$. Comparison of the oldest group of patients with the youngest subgroup ( $\leq 59$ years) revealed that older patients were significantly more often treated with endocrine therapy only (36.1 vs. $11.8 \%, \mathrm{p}=$ 0.0003 ) and significantly less often with chemotherapy containing combination regimens (30.1 vs. $53.7 \%, \mathrm{p}=$

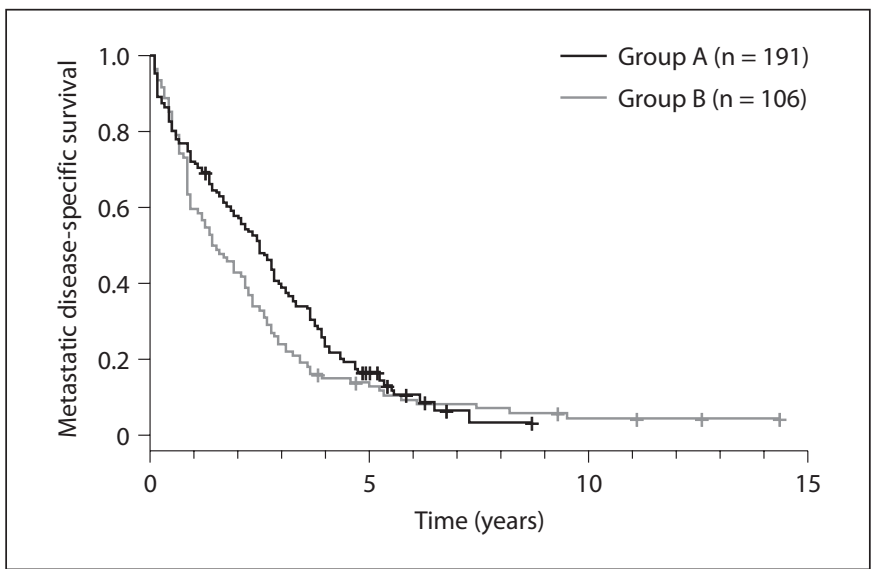

Fig. 1. MDSS among 297 patients with distant metastatic breast cancer. Group A = Distant metastases first diagnosed in 19982006; group $\mathrm{B}=$ distant metastases first diagnosed in 19901997.

$0.0001)$. In patients $>65$ years, there was no statistical difference in the percentage of patients who received chemotherapy over the course of time (1990-1997: 48.7\% vs. 1998-2006: 47.0\%; $\mathrm{p}=1.000$ ).

In the cases where chemotherapy was given, there were no differences between the number of lines administered among the three age-dependent subgroups (median: 2 lines), and in particular, there was no difference between older and younger patients ( $\geq 70$ vs. $<70$ years; $\mathrm{p}=0.269$ ). Only $26 \%$ of the patients in the current palliative setting (i.e. diagnosed with distant metastases after 1998) received more than three chemotherapy lines (table 4).

The MDSS between the study groups showed a trend towards an improved survival time in the patients whose metastatic disease was diagnosed after 1998 (fig.1; $\mathrm{p}=$ 0.062). In this group (group A), the 3 -year adjusted survival rate was $38.5 \%$ compared to group B (23.8\%) in which distant metastases were diagnosed between 1990 and 1997; the 5-year rates were 16.6 and $12.6 \%$, respectively. The median MDSS time increased from 16 months in the period from 1990 to 1997, to 21 months in the period from 1998 to 2000 .

\section{Discussion}

The outcome of patients with metastatic breast cancer varies considerably depending on host and tumor factors/ characteristics $[2,3]$. The primary goal of palliative treat- 
ment includes prevention and palliation of symptoms, maintenance or improvement of quality of life and prolongation of survival. In the palliative setting, there is currently no standard care for this heterogeneous group of patients $[3,5,6]$. Treatment decisions are made on an individual basis, and the decision-making process regarding which agents to select from the wide array of antineoplastic drugs available is complex. The choice of the therapy regimen depends on a large number of considerations [2, 3]:

- Patient-related factors: age, presence of comorbidities, physical condition, personal preferences and motivation

- Prognostic factors: interval from initial diagnosis to diagnosis of metastatic disease, rate of disease progression, number and site of metastases

- Biologic factors: hormone receptor and HER-2/neu status

- Case-related factors: type of adjuvant therapy received

- Symptom-related factors: need for rapid alleviation of severe disease-related symptoms

- Therapy-related factors: expected efficacy, type and extent of side effects, complications of prior therapy

- Physician-related factors: estimates of prognosis, experience, personal preferences towards certain agents, ability to communicate and involve the patient and her family

- Socioeconomic factors: insurance status, availability of certain agents (these factors did not play a role in our study cohort in Switzerland)

In the literature, there is a large amount of evidencebased information concerning possible therapy options. However, most of the published studies evaluate only certain therapy options in preselected groups of patients in selected situations and do not describe the overall course of metastatic disease. Decision algorithms to assist physicians in choosing appropriate therapies have been established [3]. To the best of our knowledge, our study is the first to evaluate from a population-based perspective over a fairly long period of time (1990-2006) how the available therapy options and regimens are implemented in clinical practice. Although the database from which our data were derived was created from a single primary treatment unit, the results of our study reflect the general palliative therapy situation. During the follow-up of our patients, information concerning palliative treatment was obtained from approximately 20 oncology units in Switzerland, as well as neighboring regions in Germany and France.

Systemic Therapy of Metastatic Breast Cancer
Since there has been considerable change in the last 2 decades in the number and type of agents available, we divided the patients of our study group into two subgroups according to the date of initial diagnosis of metastatic disease. As demonstrated in table 5, therapy regimens and certain drugs were used in the earlier group from 1990 to 1997 (e.g., endocrine therapy: aminoglutethimide, megestrol acetate, medroxyprogesterone acetate; chemotherapy: Leukeran, methotrexate, mitomycin C, mitoxantrone, cyclophosphamide, fluorouracil), which have mostly been replaced by modern agents and concepts. The second cohort, which consists of patients whose palliative treatment started after 1998, reflects the contemporary treatment situation with currently used agents (e.g., endocrine therapy: third-generation aromatase inhibitors, fulvestrant; chemotherapy: taxanes, capecitabine, liposomal doxorubicin, gemcitabine, vinorelbine; trastuzumab).

It was the goal of this study to depict the entire group of patients with metastatic disease in a population-based manner. In order to best achieve this, patients who received no treatment by specialized oncologists also had to be included. The number of patients who died from metastatic disease without receiving any antineoplastic therapy was surprisingly high: altogether $13.2 \%$. The lack of treatment was most commonly seen in the subgroup of older patients, since the physical condition and frequent comorbidities of these patients make oncologic therapies often impossible. However, in the subgroup of patients who were younger than 60 years, approximately $10 \%$ also did not receive any therapy before a breast cancer-related death occurred. This group of younger women rarely had any relevant comorbidities and were potential candidates for chemotherapy. This finding can be explained, excluding the few patients who had a fulminant disease course and thus could not begin timely therapy, by the fact that most patients in this group were individuals who knowingly declined therapy. Such patients are rarely ever even seen by a specialized oncologist and thus play little role in their perception of the disease. Despite the undisputed success of modern therapy approaches, a certain percentage of the population may have a profound mistrust of and aversion to modern western medicine and declines such treatment. Skepticism of more aggressive therapy regimens is also reflected in the fact that in the group of patients who died of cancer and received treatment, approximately $25 \%$ only had endocrine regimens, and of these patients, approximately $20 \%$ were younger than 60 years.

Oncology 2009;76:247-253 
Table 5. Chemotherapies, trastuzumab and endocrine therapies administered for the treatment of metastatic breast cancer

\begin{tabular}{lcc}
\hline & Group A & Group B \\
\hline Chemotherapy/trastuzumab, \% & & \\
Conventional anthracyclines & 6.7 & 19.9 \\
Liposomal doxorubicin & 9.3 & 0.8 \\
Paclitaxel & 9.3 & 2.7 \\
Docetaxel & 6.5 & 1.2 \\
Vinorelbine & 16.8 & 4.3 \\
Gemcitabine & 8.8 & 0.4 \\
Capecitabine & 15.5 & 2.7 \\
Trastuzumab & 13.2 & 0.8 \\
Cyclophosphamide & 4.9 & 13.7 \\
Methotrexate & 1.5 & 15.3 \\
5-Fluorouracil & 1.5 & 17.2 \\
Mitomycin C & 0.8 & 5.2 \\
Leukeran & 0.3 & 5.2 \\
Novantron & 2.1 & 7.8 \\
Others & 2.8 & 2.8 \\
Endocrine therapy, \% & & \\
Tamoxifen & 20.8 & 40.7 \\
Megestrol acetate & 4.1 & 20.4 \\
Aminoglutethimide & - & 21.2 \\
Third-generation aromatase inhibitor & 64.9 & 15.0 \\
Luteinizing hormone-releasing & & \\
hormone agonists & 4.1 & 2.7 \\
Fulvestrant & 6.1 & - \\
& & \\
& &
\end{tabular}

Analyses also included patients with ongoing therapies. Group $\mathrm{A}=$ Distant metastases first diagnosed in 1998-2006, $\mathrm{n}=106$; group $\mathrm{B}=$ distant metastases first diagnosed in $1990-1997, \mathrm{n}=$ 191.

In recent years, older breast cancer patients have come increasingly into the focus of interest. Today, women older than 70 years treated with chemotherapy for metastatic disease derive similar benefits as their younger counterparts [7]. In the last decade, chemotherapy in the palliative situation has changed in such a way that even older women can receive these therapy regimens. Currently, there is a selection of drugs with safer profiles, including weekly taxane regimens, newer less cardiotoxic anthracycline formulations, capecitabine, gemcitabine, and vinorelbine. In addition to new antineoplastic drugs, some advances have been made in supportive care. In our study, patients older than 70 years who qualified for chemotherapy received on average two lines of treatment, which was equal to the median number of lines administered in younger patient groups [8]. These results confirm the feasibility of chemotherapy in older patients. Surprisingly, the general readiness for treatment with chemo- therapy, probably of both the physicians and patients, did not change over time. In our study, the number of patients who were older than 70 years and received chemotherapy in the current study period (1998-2006) did not significantly differ from that in the preceding 10 -year observation period (although the agents prescribed had changed).

Our study confirms the findings and conclusions of Andre et al. [9] and Chia et al. [8] who found that an improved survival was associated with the use of newer agents and regimens over time. This finding is even more relevant when one considers that an increased use of adjuvant therapy in the later cohorts may have reduced the effectiveness of subsequent palliative systemic therapy in these patients, since a greater proportion was no longer 'therapy naive'; in this context, it should be mentioned that the increased use of adjuvant therapies, both chemoand endocrine therapies, may explain the differences in the median disease-free survival (group A: 44.5 months; group B: 25.5 months). We agree with Chia et al. that leadtime bias, such as improved diagnostic testing and change of the follow-up guidelines, did not contribute significantly to this development. Furthermore, we agree with them that improved survival cannot be attributed to a single agent or regimen. The differences in survival are most likely associated with the introduction of a new generation of agents, above all the third-generation aromatase inhibitors letrozole, anastrozole and exemestane (these agents made up 65\% of the endocrine therapies of patients whose metastases were diagnosed after 1998, while in the preceding period it was only $15 \%$ ), new chemotherapy agents and trastuzumab (taxanes, liposomal doxorubicin, vinorelbine, gemcitabine, capecitabine and trastuzumab made up approximately $80 \%$ of therapies in patients whose metastases were diagnosed after 1998, while in the preceding period it was only 13\%). The fact that there were no significant differences in either the mean or the median number of treatments delivered between the two cohorts underlines our conclusion that the use of newer agents and regimens improved survival.

Despite the progress made in palliative therapy, one should remain aware of the fact that the majority of patients have only a limited survival time of 18-24 months after the initial diagnosis of distant metastases $[8,10,11]$. In this short time period, only a limited number of therapy lines can be administered. Evidence-based medicine provides some support for the use of second-line and, to a lesser degree and in selected cases, third-line chemotherapy; beyond third-line treatment, there are no data that suggest a clear potential benefit for such a therapeu- 
tic approach [12]. In our current study cohort, $75 \%$ of the patients who received chemotherapy did not receive more than three lines of treatment.

In conclusion, we believe that this is the first study to give a population-based image of the palliative treatment situation in metastatic breast cancer and to demonstrate to what extent therapy decision algorithms were imple- mented in the clinical practice. Although metastatic breast cancer still has to be considered as an incurable disease, we support the hope that through the availability and use of newer and more effective systemic agents, the outcome of patients can be improved. This trend provides hope for further improvement in the treatment of metastatic disease in the future.

\section{References}

1 Bergh J, Jonsson PE, Glimelius B, Nygren P: A systematic overview of chemotherapy effects in breast cancer. Acta Oncol 2001;40: 253-281.

2 Chung CT, Carlson RW: Goals and objectives in the management of metastatic breast cancer. Oncologist 2003;8:514-520.

3 Ellis M, Hayes D, Lippman M: Treatment of metastatic breast cancer; in Harris J, Lippman M, Morrow M, Osborne K (eds): Diseases of the Breast. Philadelphia, Lippincott Williams \& Wilkins, 2004, pp 1101-1159.

4 Esteva FJ, Valero V, Pusztai L, Boehnke-Michaud L, Buzdar AU, Hortobagyi GN: Chemotherapy of metastatic breast cancer: what to expect in 2001 and beyond. Oncologist 2001;6:133-146.
5 Gralow JR: Optimizing the treatment of metastatic breast cancer. Breast Cancer Res Treat 2005;89(suppl 1):S9-S15.

6 Nicolini A, Giardino R, Carpi A, Ferrari P, Anselmi L, Colosimo S, Conte M, Fini M, Giavaresi G, Berti P, Miccoli P: Metastatic breast cancer: an updating. Biomed Pharmacother 2006;60:548-556.

7 Wildiers H, Kunkler I, Biganzoli L, Fracheboud J, Vlastos G, Bernard-Marty C, Hurria A, Extermann M, Girre V, Brain E, Audisio RA, Bartelink H, Barton M, Giordano SH, Muss H, Aapro M: Management of breast cancer in elderly individuals: recommendations of the International Society of Geriatric Oncology. Lancet Oncol 2007;8:1101-1115.

8 Chia SK, Speers CH, D’Yachkova Y, Kang A, Malfair-Taylor S, Barnett J, Coldman A, Gelmon KA, O'Reilly SE, Olivotto IA: The impact of new chemotherapeutic and hormone agents on survival in a population-based cohort of women with metastatic breast cancer. Cancer 2007;110:973-979. $\checkmark 9$ Andre F, Slimane K, Bachelot T, Dunant A, Namer M, Barrelier A, Kabbaj O, Spano JP, Marsiglia H, Rouzier R, Delaloge S, Spielmann M: Breast cancer with synchronous metastases: trends in survival during a 14year period. J Clin Oncol 2004;22:33023308.

$>10$ Fossati R, Confalonieri C, Torri V, Ghislandi E, Penna A, Pistotti V, Tinazzi A, Liberati A: Cytotoxic and hormonal treatment for metastatic breast cancer: a systematic review of published randomized trials involving 31,510 women. J Clin Oncol 1998;16:34393460 .

11 Stockler M, Wilcken NR, Ghersi D, Simes RJ: Systematic reviews of chemotherapy and endocrine therapy in metastatic breast cancer. Cancer Treat Rev 2000;26:151-168.

12 Cardoso F, Di LA, Lohrisch C, Bernard C, Ferreira F, Piccart MJ: Second and subsequent lines of chemotherapy for metastatic breast cancer: what did we learn in the last two decades? Ann Oncol 2002;13:197-207. 